\title{
Redox-Chemistry of Environmental Biofilms Probed on the Submicron Scale by in- situ Electrochemistry Scanning Transmission (soft) X-ray Microscopy
}

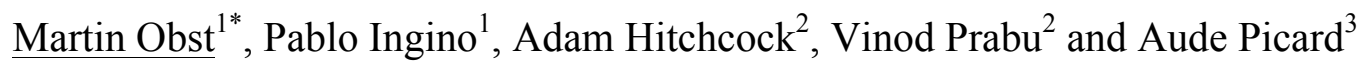 \\ 1. Experimental Biogeochemistry, BayCEER, University of Bayreuth, Bayreuth, Germany \\ 2. Department of Chemistry and Chemical Biology, McMaster University, Hamilton, ON, Canada \\ 3. Harvard University, Department of Organismic and Evolutionary Biology, Cambridge, MA, USA \\ * Corresponding author, martin.obst@uni-bayreuth.de
}

Introduction: A complex interplay of (redox-)chemical and biological processes control the fate of nutrients and contaminants in the environment. These can only be understood when the underlying reaction mechanisms can be identified and quantified. However, there is an increasing awareness of heterogeneity on various spatial and temporal scales in biogeochemical processes. Temporal fluctuations, caused for example by daily or seasonal cycles, water table fluctuations or perturbations, can result in fluctuations of biogeochemical reaction zones [1,2] and thus result in unexpected reactions.

On macroscopic spatial scales various "cryptic sulfur cycles" have been described. A linkage of the S, $\mathrm{Fe}$ and $\mathrm{C}$ redox cycling was found to explain the unexpected presence of sulfate in marine sediments, far below the main sulfate zone [3]. Very recently, Hansel et al. unexpectedly demonstrated the existence of such coupling between the Fe and the S-cycle for low-sulfate freshwater environments [4]. Recently socalled cable-bacteria have been identified in sediments that are capable of conducting electrons over distances of millimeters and thus link otherwise independent oxidation and reduction reactions [5]. However, the role of microbial, supposedly redox-active, and eventually conductive structures, in mediating redox-reactions in the environment on the micron-scale is not yet understood.

We hypothesize that microorganisms present in biofilms of high-redox activity produce structures that can mediate redox-reactions and eventually contribute to electron conductivity. To verify this hypothesis, approaches are being developed that allow for identifying and quantifying redox-active organic functional groups under close to natural, hydrated conditions, and to quantify their contributions to oxidation and reduction reactions by electrochemical manipulation in combination with a spectromicroscopic characterization. In-situ electro-chemical scanning transmission (soft) X-ray

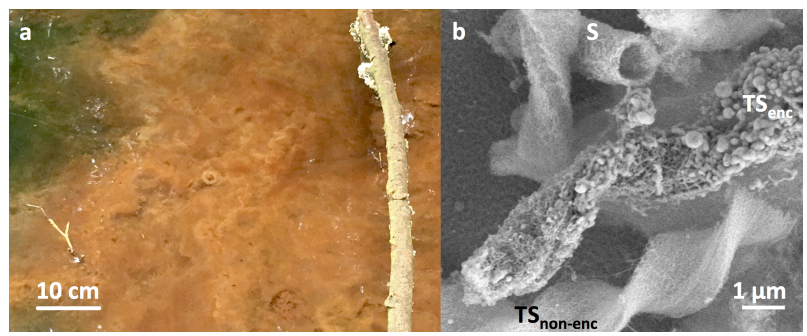

Fig. 1. a) Redox-active environmental biofilm in a creek in an abandoned, medieval mining area in the 'Fichtelgebirge' mountains, Germany. b) SEM image of microbial exopolymer structures in this biofilm: Twisted stalks, both encrusted ( $\left.\mathrm{TS}_{\mathrm{enc}}\right)$ and non-encrusted ( $\left.\mathrm{TS}_{\text {non-enc }}\right)$ in Fe-oxyhydroxides, and tubular sheaths (S) are abundant in these biofilms. microscopy (STXM) [6] has these capabilities and was adapted and optimized for its first application on microbial exopolymers.

Methods: Various supposedly redox-active environmental biofilms that are abundant in large amount in mine-drainage (e.g., Fig. 1a), were sampled in the mountainous regions "Black Forest" and "Fichtelgebirge" in Germany. These biofilms are mostly composed of microbial polymer structures such as twisted stalks and sheaths (Fig 1b) that are sometimes closely associated with iron-oxyhydroxides. To identify appropriate structures, subsamples of the biofilms were dried onto Formvar- 
coated 300-mesh $\mathrm{Cu}$ TEM grids and analyzed using the ambient STXM on Canadian Light Source (CLS) beamline 10ID-1. C 1s image sequences (stacks) of individual structures such as twisted stalks or sheaths (Fig 1b) were acquired with an energy resolution of $0.1 \mathrm{eV}$ in the energy region of interest and a pixel spacing of 20-80 nm depending on the size of the structures and regions of interest. The datasets were converted from transmission into linear absorbance (optical density, OD) scale using aXis2000 [7]. $\mathrm{C} 1 \mathrm{~s}$ spectra were decomposed into sets of an arctan step function and a number of individual Gaussian peaks with transition energies associated with specific organic functional groups using Athena [8]. The relative contributions of these moieties were quantified and compared among different samples. Redoxactive functional moieties such as quinone or phenolic groups were of particular interest.

To quantify the contributions of these moieties to the redox-activity of the organic structures, a combination of electrochemical manipulation and spectromicroscopic characterization was used, that is currently under ongoing development. Samples were analyzed in sandwich wet cells prepared from a $\mathrm{Si}_{3} \mathrm{~N}_{4}$ window equipped with gold electrodes capped with a second $\mathrm{Si}_{3} \mathrm{~N}_{4}$ window (Norcada Inc., Edmonton, AB, Canada). A custom potentiostat setup, based on a National Instruments MyDAQ (NI, Austin TX, US) and a low current amplifier (FEMTO GmbH, Berlin, Germany) was used. In a second step, dissolved redox-active model compounds such as quinhydrone will be analyzed in a newly developed microfluidic flow-through electrochemical STXM setup using $\mathrm{Si}_{3} \mathrm{~N}_{4}$ windows equipped with gold electrodes and a spacer chip $\mathrm{Si}_{3} \mathrm{~N}_{4}$ window with a microfluidic flow channel (Norcada), using a miniature potentiostat (Pocketstat, Ivium Technologies, Eindhoven, The Netherlands).

Results \& Discussion: To quantify the fractions of redox-active carbon and $\mathrm{C}$ that is independent of the applied potential, the average spectra extracted at three different potentials were used for linear fits. The resulting maps of "oxidized" and "reduced" species were masked and used to extract improved spectra from these regions showing reduced, original or oxidized characteristics. The resulting spectra were then normalized to $1 \mathrm{~nm}$ layer of organic carbon and used for quantitative mapping.

Individual microbial polymers structures such as twisted stalks (Fig. 2a) were analyzed spectromicroscopically at the $\mathrm{C} 1 \mathrm{~s}$ edge. The decomposition of the resulting average spectra of individual structures (Fig. 2b) allowed for the quantification of the relative contributions of individual organic functional groups to the overall $\mathrm{C} 1 \mathrm{~s}$ spectra and for a comparison between individual structures from different sites and biogeochemical settings. Fig. 2c illustrates a comparison of the functional group composition of two structures from different environmental biofilms and a laboratory culture.

Based on these results, selected samples were analyzed by in-situ flow electrochemical STXM [6]. Individual stalks could be placed on the electrode of the e-chem STXM setup (Fig. 2d). Spectra, recorded while potentials of $-0.5 \mathrm{~V}, 0.0 \mathrm{~V}$, and $+0.5 \mathrm{~V}$ were applied, show significant differences at energies specific for quinone-C $(\sim 284.0 \mathrm{eV})$, phenolic $\mathrm{C}(\sim 286.6 \mathrm{eV})$ but also large changes at higher energies that are not specific to individual chemical bonds. When the electrode acts as a cathode $(+0.5$ $\mathrm{V}$ ), the absorption specific for quinone-C is lower, and the phenolic-C absorption rises (arrows in Fig. $2 \mathrm{e})$. When no potential is applied $(0.0 \mathrm{~V})$, and when the electrode acts as an anode $(-0.5 \mathrm{~V})$, the quinone$\mathrm{C}$ absorption is higher.

Quantification of redox-active fractions of the organic structures is shown in Fig. 2f. This analysis is based on linear combination fitting with spectra that were iteratively extracted from the datasets recorded at three different potentials. The results show unambiguously a significant reduction at a 
potential of $+0.5 \mathrm{~V}$ whereas the oxidation at $-0.5 \mathrm{~V}$ is less pronounced. We conclude that the stalk analyzed by in-situ e-chem STXM shows higher redox-activity and redox-capacity under reducing conditions as compared to oxidizing conditions.
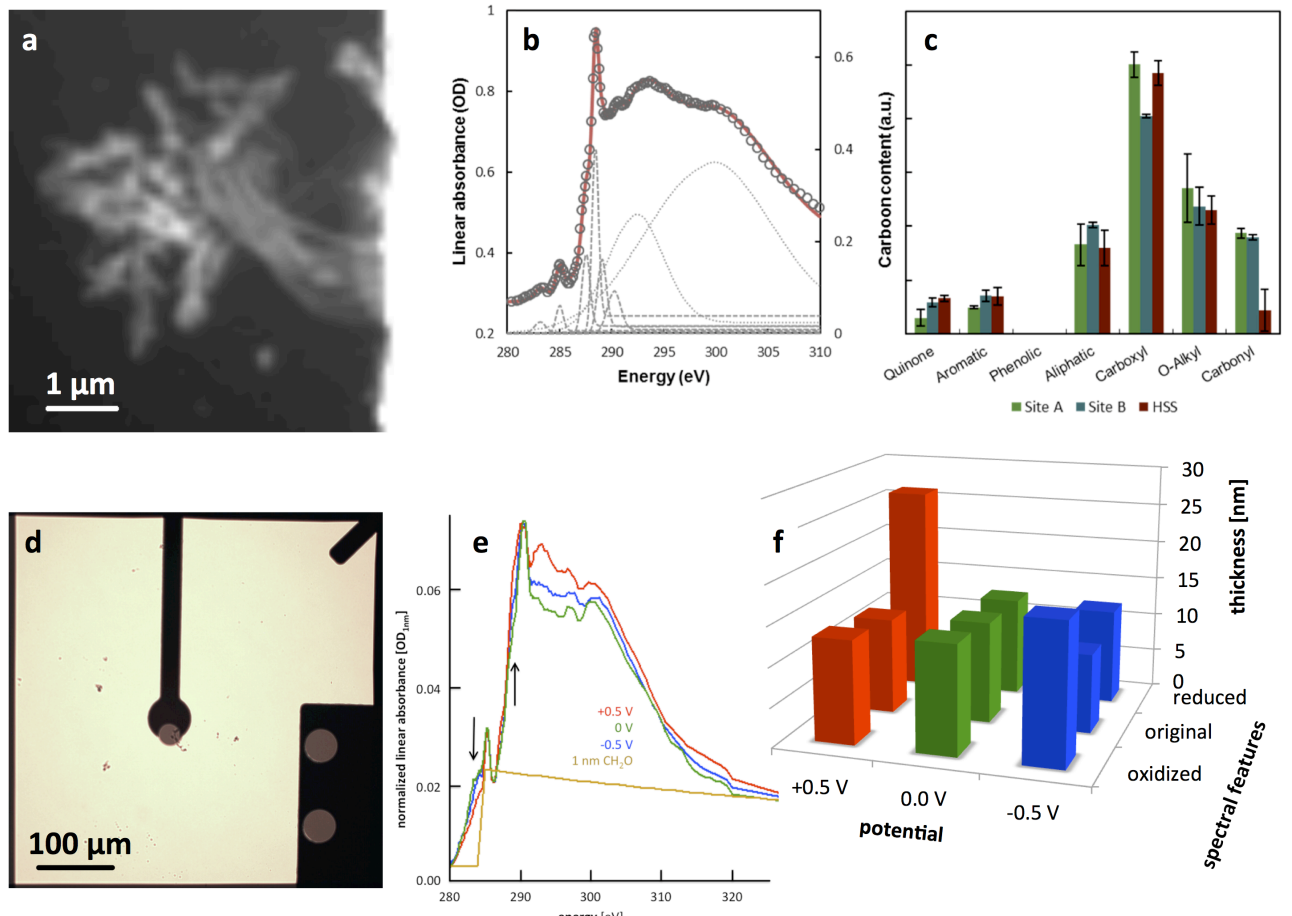

Figure. 2. a) C 1s OD-image of an individual twisted stalk. b) Spectrum of an individual structure, decomposed into individual peaks of certain functional moieties. c) Relative quantification of these moieties for comparison between different microbial organic structures. d) Light microscopy image of in-situ electrochemical STXM cell with a 3-electrode setup; a twisted stalk is attached to the working electrode in the center. e) Normalized spectra, acquired at different potentials; arrows indicate spectral changes at the energies of quinone-C and phenolic-C. f) Iteratively extracted spectra recorded at different potentials were used to quantify the total amount of $\mathrm{C}$ that is influenced by oxidation or reduction (i.e. spectral features on y-axis).

\section{References:}

[1] G.W. Luther, et al., Mar Chem 40 (1992), 81.

[2] P.T. Ha, et al., Front Microbiol 6 (2015), 909, http://doi.org/10.3389/fmicb.2015.00909

[3] L Holmkvist, et al., Geochim Cosmochim Acta 75 (2011) 3581.

[4] C.M. Hansel, et al., ISME J 9 (2015) 2400.

[5] L.P. Nielsen, et al., Nature 463 (2010) 1071.

[6] V. Prabu et al., Rev. Sci. Inst. (2018) in press.

[7] A.P. Hitchcock (March 2018), aXis2000 written in IDL, available free for non-commercial use from http:// unicorn.mcmaster.ca/aXis2000.html

[8] B. Ravel \& M. Newville, J. Synchrotron Radiat 12 (2005) 537

[9] We thank I. Adaktylou, F. Zeitvogel and G. Schmid for discussion and help during preliminary experiments. We thank J. Wang, Y. Lu, J. Geilhufe and J. Dynes (CLS) for their valuable help. Research was performed at CLS, which is supported by the Canada Foundation for Innovation. We also acknowledge DFG funding through the Heisenberg program (DFG grant OB 362/4-1). 
https://doi.org/10.1017/S1431927618014745 Published online by Cambridge University Press 